\title{
MEASUREMENT OF PARAMETERS OF TRACKS IN CR-39 DETECTOR FROM REPLICAS
}

\author{
K. N. Yu*, F. M. F. Ng, J. P. Y. Ho, C. W. Y. Yip and D. Nikezic \\ Department of Physics and Materials Science, City University of Hong Kong, Tat Chee Avenue, \\ Kowloon Tong, Kowloon, Hong Kong, Peoples Republic of China
}

\begin{abstract}
In determining the etched track rate in solid-state nuclear track detectors, track lengths should be determined accurately. A method based on surface profilometry is proposed to determine the track lengths in CR-39 detectors through measurements of their replicas. Tracks from alpha particles with an incident energy of $4 \mathrm{MeV}$ have been chosen to demonstrate the method. After irradiation and chemical etching, resin replicas were made from the tracks, of which the heights were measured by the Form Talysurf PGI Profilometer. The results showed that the surface of the replicas were smooth and the heights of the replicas were uniform, so the replicating fluid should have filled the tracks completely and the replicas truly reflected the dimensions of the tracks. The heights of the replicas were conveniently determined from the lateral view of the replicas generated by the Form Talysurf PGI Profilometer.
\end{abstract}

\section{INTRODUCTION}

Solid-state nuclear track detectors (SSNTDs) have found applications in different branches of science. Many researches have been devoted to understanding the mechanisms of track growth in SSNTDs. The most widely accepted track growth model involves two etch rates, namely, the etched track rate $V_{\mathrm{t}}$ (i.e. along a track in the SSNTD) and the bulk etch rate $V_{\mathrm{b}}$ (i.e. in undamaged areas of the SSNTD). All proposed methods for track growth should be tested with experimental data including, e.g. the track-opening diameters and the track lengths.

Measurements of these alpha track parameters are usually performed by optical methods. Although measurements of track-opening diameters are relatively straightforward, direct measurements of track lengths are relatively difficult. One approach involves the breaking of SSNTDs to reveal the lateral images of the tracks for direct measurements ${ }^{(1)}$ and another involves the use of confocal microscopy $^{(2)}$. In the present work, we propose a new method based on surface profilometry to determine the lengths of tracks in CR-39 detectors through measurements of their replicas. The replicas are required because of the geometry of the stylus of the surface profilometry equipment which may prevent the stylus from reaching the bottom of the tracks.

\section{METHODOLOGY}

For the sake of demonstrating the applicability of surface profilometry in revealing the lengths of alpha tracks in the CR-39 detectors, only alpha particles with an incident energy of $4 \mathrm{MeV}$ and with normal

${ }^{*}$ Corresponding author: peter.yu@cityu.edu.hk incidence on the detectors are studied. The CR39 detectors used in the present study were purchased from Page Mouldings (Pershore) Limited (Worcestershire, England). The original dimensions of a sheet of the detector are $30 \times 47 \times 0.1 \mathrm{~cm}$ (thickness). The detectors for our studies were cut to a size of $1.5 \times 1.5 \mathrm{~cm}^{2}$.

The CR-39 detectors were irradiated with alpha particles with an energy of $4 \mathrm{MeV}$ under normal incidence through a collimator. The alpha source employed in the present study was a planar ${ }^{241} \mathrm{Am}$ source (main alpha energy $=5.49 \mathrm{MeV}$ under vacuum). Normal air was used as the energy absorber to control the final alpha energies incident on the detector. A relationship between the alpha energy and the air distance travelled by an alpha particle (with initial energy of $5.49 \mathrm{MeV}$ from ${ }^{241} \mathrm{Am}$ ) was therefore needed. This relationship was obtained by measuring the energies for alpha particles passing different distances through normal air using alpha spectroscopy systems (ORTEC Model 5030) with Passivated Implanted Planar Silicon (PIPS) detectors of areas of $300 \mathrm{~mm}^{2}$.

After irradiation, the detectors were etched in a $6.25 \mathrm{~N}$ aqueous solution of $\mathrm{NaOH}$ maintained at $70^{\circ} \mathrm{C}$ in a water bath for $15 \mathrm{~h}$, which was the normally employed etching time. The detectors were then taken out from the etchant, rinsed with distilled water and dried in air. A plastic mould in the form of a cylindrical cup with a detachable bottom was used to prepare the resin replicas using Buehler fast cure epoxy (41 Waukegan Rd, Lake Bluff, IL 60044, USA). The inside surface of the mould was first coated with the Buehler Release Agent (No. 208185-002). A piece of etched detector (with the alpha tracks) was then placed inside the mould on the bottom, with the side containing the tracks facing upwards. The replicating fluid was prepared with Buehler Epo-Kwick resin (No. 20-8136-128) 


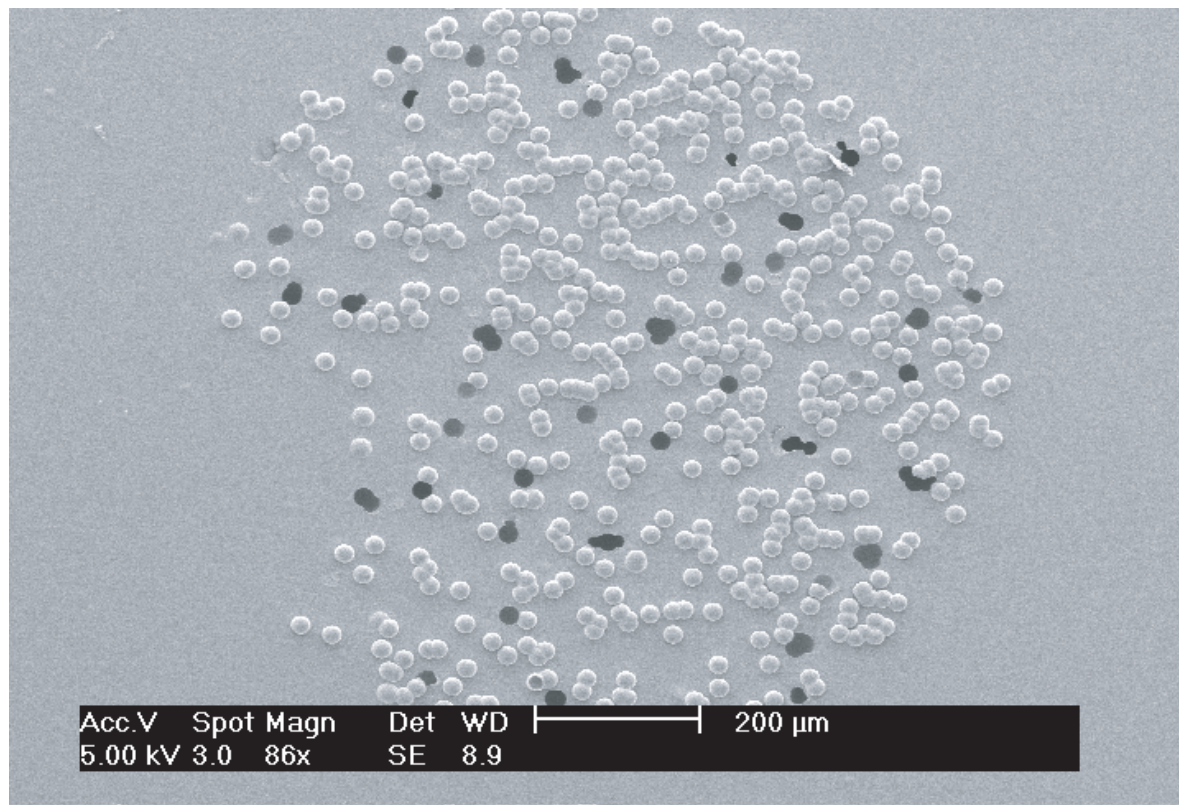

Figure 1. Three-dimensional SEM image (viewing directly from above) of replicas of tracks resulted from normally incident $4 \mathrm{MeV}$ alpha particles and $15 \mathrm{~h}$ etching.

and Buehler Epo-Kwick hardener (No. 20-8138-032) with the mass ratio of $5: 1$, and was poured into the mould. After drying for more than $10 \mathrm{~h}$, the mould was detached. The CR-39 detector was then removed from the resin after sawing along some edges of the CR-39 detector, leaving behind the replicas of the tracks protruding from the resin surface.

The track lengths were then measured by the Form Talysurf PGI Profilometer (Taylor Hobson, Leicester, England), which is a contact stylus instrument based on a Phase Grating Interferometric (PGI) transducer. The PGI transducer is within a gauge and converts the movements of the stylus into an electrical signal. This type of transducer employs a laser diode in conjunction with a fine optical grating to detect very small movements of the stylus and provides a high-resolution output of both amplitude and direction. During measurements, the stylus was set to scan the studied surface many times to ensure that the stylus would pass across any one track at least 10 times.

\section{RESULTS AND DISCUSSION}

Figure 1 shows the three-dimensional (3D) SEM image (viewing directly from above) of replicas of tracks resulted from normally incident $4 \mathrm{MeV}$ alpha particles and $15 \mathrm{~h}$ etching. Figure 2 shows the 3D SEM image of the same replicas with the stage inclined at $40^{\circ}$. From Figures 1 and 2, we can see that the surfaces of the replicas are smooth and the heights of the replicas are uniform (the latter also observed from surface profilometry measurements). Therefore, we are confident that the replicating fluid has filled the tracks completely and the replicas can truly reflect the dimensions of the tracks. This is also proved by the optical microscopic image shown in Figure 3 showing the cross sections of the tracks with the dried replicating fluid inside. The replicas of the tracks are shown clearly, and the replicating fluid is shown to fill the tracks completely.

Figure 4 shows the (2D) image of the replicas recorded by the Form Talysurf PGI Profilometer. The dimensions as well as the lengths of the tracks are conveniently read from the figure. Again, we can see from Figure 4 that the heights of the replicas are very uniform. The Form Talysurf PGI Profilometer can also generate the lateral view of the replicas. An example is shown in Figure 5. It is even easier to determine the heights of the replicas from this view. From Figure 5, the heights of the replicas are $16 \mu \mathrm{m}$. The theoretical range of $4 \mathrm{MeV}$ alpha particles in the CR-39 detector is $20.75 \mu \mathrm{m}$. The $V_{\mathrm{b}}$ of the present etching was $1.2 \mu \mathrm{m} \mathrm{h}^{-1(3)}$. With this $V_{\mathrm{b}}$ and the $V_{\mathrm{t}}$ function given by Durrani and Bull ${ }^{(4)}$, the track development model of Nikezic and $\mathrm{Yu}^{(5)}$ gave the track length as $15.34 \mu \mathrm{m}$, which agrees with the experimental value. 


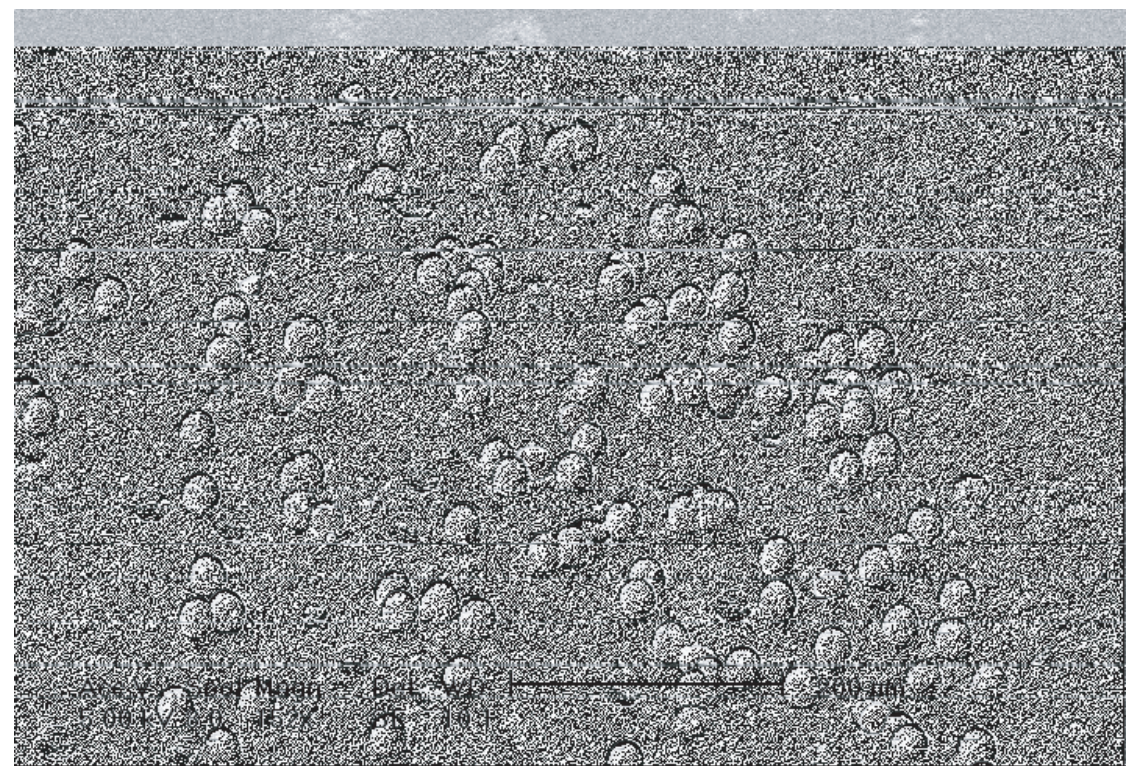

Figure 2. Three-dimensional SEM image (with the stage inclined at $40^{\circ}$ ) of replicas of tracks resulted from normally incident $4 \mathrm{MeV}$ alpha particles and $15 \mathrm{~h}$ etching.

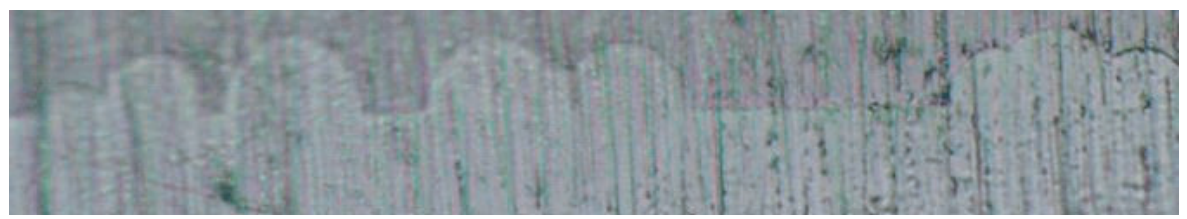

Figure 3. Optical microscopic image showing the cross sections of the tracks with the dried replicating fluid inside. The upper part is the detector while the lower part is the replicating fluid. The replicas of the tracks are shown clearly.

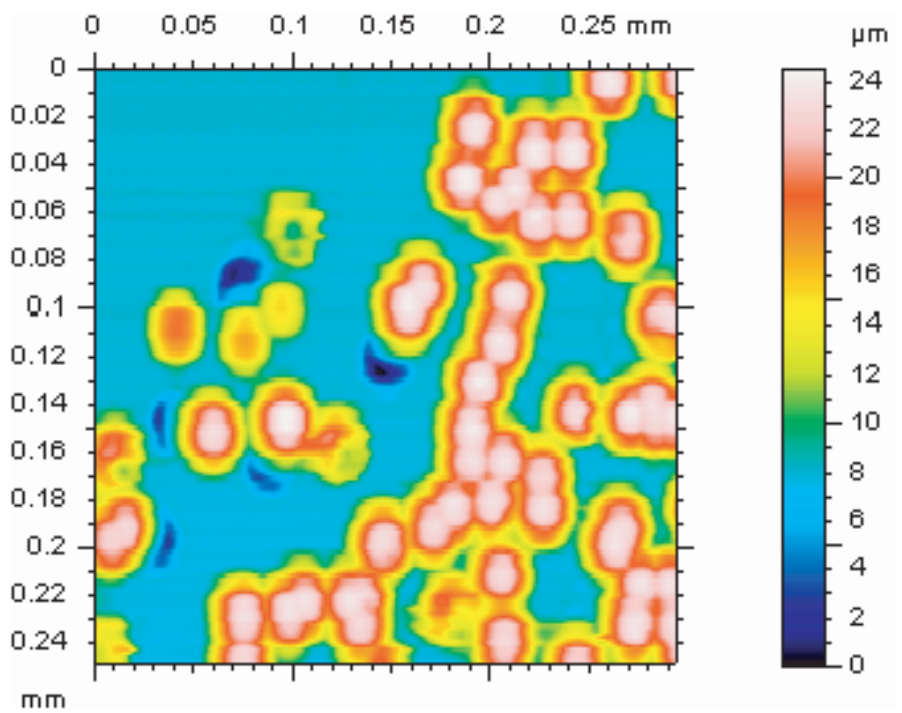

Figure 4. Two-dimensional image of the replicas recorded by the Form Talysurf PGI Profilometer. 


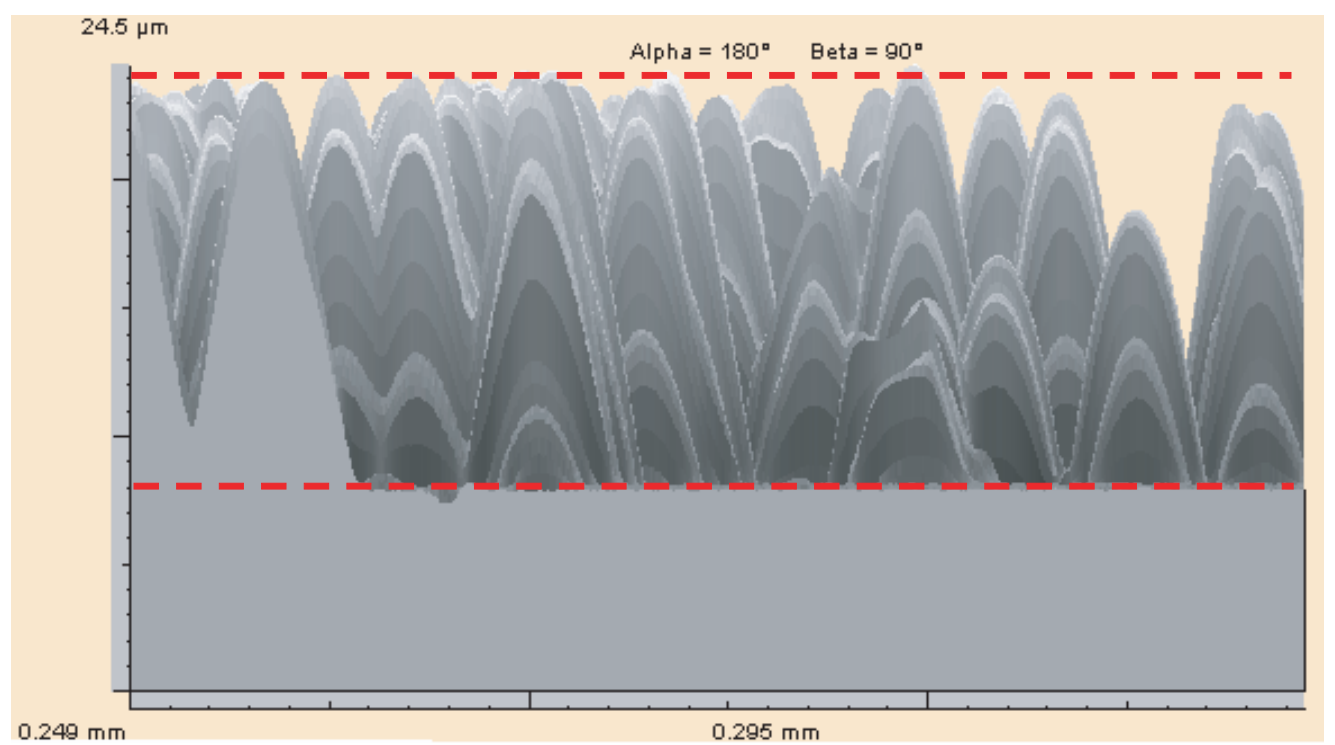

Figure 5. Lateral view of the replicas generated by the Form Talysurf PGI Profilometer. The typical height of the replicas is given by the difference between the dotted lines, which is $16 \mu \mathrm{m}$.

The above procedures have demonstrated the convenience to measure the track lengths by surface profilometry measurements using replicas of the tracks. As protruding objects are being measured, the track lengths will not be distorted due to artefacts produced by the geometry of the stylus of the surface profilometry equipment. To obtain the required $V_{\mathrm{t}}$ function, replicas from tracks formed by alpha particles with different incident energies have to be measured.

\section{ACKNOWLEDGEMENTS}

The present research is supported by the CERG grant CityU 102803 from the Research Grant Council of Hong Kong.

\section{REFERENCES}

1. Dörschel, B., Fülle, D., Hartmann, H., Hermsdorf, D., Kadner, K. and Radlach, Ch. Measurement of track parameters and etch rates in proton-irradiated CR-39 detectors and simulation of neutron dosemeter responses. Radiat. Prot. Dosim., 69(4), 267-274 (1997).

2. Vaginay, F., Fromm, M., Pusset, D., Meesen, G., Chambaudet, A. and Poffijn, A. 3-D confocal microscopy track analysis: a promising tool for determining CR-39 response function. Radiat. Meas., 34, 123-127 (2001).

3. Ho, J. P. Y., Yip, C. W. Y., Nikezic, D. and Yu, K. N. Effects of stirring on the bulk etch rate of CR-39 detector. Radiat. Meas., 36, 141-143 (2003).

4. Durrani S. A. and Bull, R. K. Solid State Nuclear Track Detection: Principles, Methods and Applications. (Oxford: Pergamon Press) (1987).

5. Nikezic, D. and Yu, K. N. Three-dimensional analytical determination of the track parameters. Over-etched tracks. Radiat. Meas., 37, 39-45 (2003). 